\title{
PROPERTIES OF $T$-SPREAD PRINCIPAL BOREL IDEALS GENERATED IN DEGREE TWO *
}

\author{
Bahareh Lajmiri and Farhad Rahmati
}

(c) 2020 by University of Niš, Serbia | Creative Commons License: CC BY-NC-ND

\begin{abstract}
In this paper, we have studied the stability of $t$-spread principal Borel ideals in degree two. We have proved that $\operatorname{Ass}^{\infty}(I)=\operatorname{Min}(I) \cup\{\mathfrak{m}\}$, where $I=B_{t}(u) \subset S$ is a $t$-spread Borel ideal generated in degree 2 with $u=x_{i} x_{n}, t+1 \leq i \leq n-t$. Indeed, $I$ has the property that $\operatorname{Ass}\left(I^{m}\right)=\operatorname{Ass}(I)$ for all $m \geq 1$ and $i \leq t$, in other words, $I$ is normally torsion free. Moreover, we have shown that $I$ is a set theoretic complete intersection if and only if $u=x_{n-t} x_{n}$. Also, we have derived some results on the vanishing of Lyubeznik numbers of these ideals.

Keywords: Monomial ideals, t-spread principal Borel ideals, Arithmetical rank, Complete intersection.
\end{abstract}

\section{Introduction}

Let $S=K\left[x_{1}, \ldots, x_{n}\right]$ be a polynomial ring and $I \subset S$ a graded ideal. By a wellknown result of Brodmann [4], there exists an integer $k \geq 1$ such that $\operatorname{Ass}\left(I^{m}\right)=$ $\operatorname{Ass}\left(I^{k}\right)$ for all $m \geq k$. A prime ideal $P \in \operatorname{Ass}^{\infty}(I)=\bigcup_{m \geq 1} \operatorname{Ass}\left(I^{m}\right)$ is called persistent with respect to $I$, and whenever $P \in \operatorname{Ass}\left(I^{k}\right)$ we have $P \in \operatorname{Ass}\left(I^{k+1}\right)$. The ideal $I$ has the persistence property if all the prime ideals $P \in \operatorname{Ass}^{\infty}(I)$ are persistent, that is, if $\operatorname{Ass}(I) \subseteq \operatorname{Ass}\left(I^{2}\right) \subseteq \cdots \subseteq \operatorname{Ass}\left(I^{m}\right) \subseteq \cdots$.

The persistence property for monomial ideals has been intensively studied in the last years; see for example, [10] and the references therein. Recently, it has been proved in [1] that $t$-spread principal Borel ideals have the persistence property. The so-called $t$-spread ideals were introduced in [7].

Let $t \geq 1$ be an integer. A monomial $x_{i_{1}} \cdots x_{i_{d}} \in S$ with $i_{1} \leq \cdots \leq i_{d}$ is called $t$-spread if $i_{j}-i_{j-1} \geq t$ for $2 \leq j \leq d$. We recall from [7] that a monomial ideal $I \subset S$ with the minimal system of monomial generators $G(I)$ is called $t$-spread principal Borel if there exists a monomial $u \in G(I)$ such that $I=B_{t}(u)$, where $B_{t}(u)$ denotes the smallest $t$-spread strongly stable ideal which contains $u$. A monomial ideal $I$ is

Received January 5, 2019, accepted November 24, 2019

2010 Mathematics Subject Classification. Primary D13D02; Secondary13H10, 05E40, 13C14

${ }^{*}$ The authors were supported in part by ... 
called $t$-spread strongly stable if it satisfies the following condition: for all $u \in G(I)$ and $j \in \operatorname{supp}(u)$, if $i<j$ and $x_{i}\left(u / x_{j}\right)$ is $t$-spread, then $x_{i}\left(u / x_{j}\right) \in I$.

In this paper, we will study several properties of $t$-spread principal Borel ideals $B_{t}(u)$ generated in small degree. Most part of the paper is devoted to the study of $\operatorname{Ass}^{\infty}\left(B_{t}(u)\right)$. In the second part of the paper we will study the arithmetical rank of $B_{t}(u)$. In the last part, we will derive some results on the vanishing of Lyubeznik numbers of $B_{t}(u)$.

The main result of the first section shows that if $I=B_{t}(u) \subset S$ is a $t$-spread Borel ideal generated in degree 2 with $u=x_{i} x_{n}, t+1 \leq i \leq n-t$, then $\operatorname{Ass}\left(I^{m}\right)$ is already stabilized at $m=2$ and $\operatorname{Ass}^{\infty}(I)=\operatorname{Min}(I) \cup\{\mathfrak{m}\}$, where $\operatorname{Min}(I)$ denotes the set of minimal prime ideals of $I$ and $\mathfrak{m}$ is the maximal graded ideal of $S$. The hypothesis $i \geq t+1$ might look restrictive, but as we explain in Remark 2.4, this is the only case when $\operatorname{Ass}^{\infty}(I) \supsetneq \operatorname{Min}(I)$.

For the proof, one has to consider monomial localization of a monomial ideal. Let $P=P_{A}=\left(x_{j}: j \notin A\right)$ be a monomial prime ideal and $I \subset S$ a monomial ideal. Then the localization of $I$ with respect to $P$ is $I(P) \subset S(P)=K\left[\left\{x_{j}: j \notin A\right\}\right]$ which is obtained from $I$ by applying the $K$-algebra homomorphism $S \rightarrow S(P)$ induced by $x_{j} \mapsto 1$ for $j \notin A$. Moreover, by [11, Lemma 2.3], we have $P \in \operatorname{Ass}(I)$ if and only if $\operatorname{depth} S(P) / I(P)=0$.

It was observed in [1] that all the powers of a $t$-spread principal Borel ideal have linear quotients with respect to the decreasing lexicographic order. By monomial localization of a $t$-spread principal Borel ideal generated in degree 2, we can get monomial ideals which still have linear quotients though they are not generated in a single degree. Therefore, we can compute the depth of their powers by using the projective dimension formula given in [9, Chapter 8]. Namely, let $I \subset S$ be a monomial ideal with $G(I)=\left\{u_{1}, \ldots, u_{m}\right\}$. We say that $I$ has linear quotients with respect to the order $u_{1}, \ldots, u_{m}$ of its minimal monomial generators if for every $j \geq 1$, the ideal quotient $L_{j}=\left(u_{1}, \ldots, u_{j-1}\right): u_{j}$ is generated by variables. If $r_{j}$ is the number of variables which generate $L_{j}$ for every $j$, then $\operatorname{proj} \operatorname{dim} S / I=$ $\max \left\{r_{1}, \ldots, r_{m}\right\}+1$, hence

$$
\operatorname{depth} S / I=n-1-\max \left\{r_{1}, \ldots, r_{m}\right\} .
$$

We should note that the persistence property of every $t$-spread principal Borel ideal $B_{t}(u)$ generated in degree 2 may be derived by using [6, Theorem 2.15] since $B_{t}(u)$ can be viewed as the edge ideal of a graph.

Let $I \subset S$ be a homogeneous ideal and $\sqrt{I}$ the radical of $I$. Then the arithmetical rank of $I$ is defined as

$\operatorname{ara}(I)=\min \left\{r \geq 1:\right.$ there exists $f_{1}, \ldots, f_{r} \in I$ such that $\left.\sqrt{I}=\sqrt{\left(f_{1}, \ldots, f_{r}\right)}\right\}$.

It is known that for every squarefree monomial ideal $I \subset S$, we have

$$
\operatorname{ara}(I) \geq \operatorname{cd}(I)=\operatorname{proj} \operatorname{dim}(S / I),
$$


where $\operatorname{cd}(I)$ denotes the cohomological dimension of $I$ [14].

If height $(I)=\operatorname{ara}(I)$, the ideal $I$ is called a set-theoretic complete intersection. An ideal $I$ is called cohomologically complete intersection if $h t(I)=c d(I)$.

There are several classes of squarefree monomial ideals for which equality holds in inequality (1.2); see, for example, $[3,5,8,12]$. In [12] and [5] it was shown that if $I \subset S$ is a squarefree monomial ideal with a 2-linear resolution, then $\operatorname{ara}(I)=$ $\operatorname{proj} \operatorname{dim}(S / I)$. As a consequence of [7, Theorem 1.4], it follows that every $t$-spread principal Borel ideal has a 2-linear resolution, thus if $I=B_{t}(u)$ where $u$ is a $t$-spread monomial of degree 2 , then we have $\operatorname{ara}(I)=\operatorname{proj} \operatorname{dim}(S / I)$. In Section 3. we give a direct proof of this equality by using the Schmitt-Vogel Lemma (see [15]) which might be interesting for the reader. In particular, we derive that $I=B_{t}(u)$ is a set theoretic complete intersection ideal if and only if $u=x_{n-t} x_{n}$.

Finally, in Section 4., we derive some results on the vanishing of Lyubeznik numbers of $t$-spread principal Borel ideals in degree two.

\section{Stability for the associated primes}

In this section, we aim at proving the following:

Theorem 2.1. Let I be a t-spread principal Borel ideal, where $u=x_{i} x_{n}, t+1 \leq$ $i \leq n-t$. Then

$$
\operatorname{Ass}\left(I^{m}\right)=\operatorname{Min}(I) \cup\{\mathfrak{m}\}, \text { for } m \geq 2 .
$$

In particular,

$$
\operatorname{Ass}^{\infty}(I)=\operatorname{Min}(I) \cup\{\mathfrak{m}\}
$$

In order to prove this theorem, we need some preparation.

Let $u=x_{i} x_{n}$ with $i \leq t$ and $I=B_{t}(u)$. We set $\mathcal{S}(I)=\bigcup_{v \in G(I)} \operatorname{supp}(v)$. If $i<t$, then $\mathcal{S}(I) \subsetneq[n]$. Then, as it was observed in the proof of [1, Theorem 3.1], since $I$ satisfies the $l$ - exchange property, it follows that $I^{m}$ has linear quotients with respect to $>_{\text {lex }}$ for every $m \geq 1$. This means that if $G\left(I^{m}\right)=\left\{u_{1}>_{\text {lex }} u_{2}>_{\text {lex }} \ldots u_{q}>_{\text {lex }}\right\}$ then for every $j \geq 1$, the ideal quotient $\left(u_{1}, \ldots, u_{j-1}\right): u_{j}$ is generated by variables.

Lemma 2.2. In the above settings, for every $j \geq 1, x_{n}, x_{i} \notin\left(u_{1}, \ldots, u_{j-1}\right): u_{j}$.

Proof. Clearly $x_{n} \notin\left(u_{1}, \ldots, u_{j-1}\right): u_{j}$ since we cannot write $x_{n} u_{j}$ as a multiple of $u_{l}$ with $l \leq j-1$.

As $i \leq t$, the generators of $I$ are the form of $x_{i_{l}} x_{j_{l}}$ with $1 \leq i_{l} \leq i \leq t$, $j_{l}>t$. Assume that there exists $j \geq 2$ such that $x_{i} u_{j} \in\left(u_{1}, \ldots, u_{j-1}\right)$. Let $u_{j}=$ $\left(x_{i_{1}} x_{j_{1}}\right) \ldots\left(x_{i_{m}} x_{j_{m}}\right)$ with $1 \leq i_{1} \leq i_{2} \leq \cdots \leq i_{m} \leq i \leq t$ and $t<j_{1}, \ldots, j_{m} \leq n$. Then $u_{j}=\left(x_{i_{1}} \ldots x_{i_{m}}\right)\left(x_{j_{1}} \ldots x_{j_{m}}\right)$. If $x_{i} u_{j} \in\left(u_{1}, \ldots, u_{j-1}\right)$, then there exists some monomial $u_{l} \in G\left(I^{m}\right)$ with $l \leq j-1$ such that $x_{i} u_{j}=u_{l} x_{s}$, for some $s>i$. Let $u_{l}=$ $\left(x_{i_{1}^{\prime}} \ldots x_{i_{m}^{\prime}}\right)\left(x_{j_{1}^{\prime}} \ldots x_{j_{m}^{\prime}}\right)$ with $1 \leq i_{1}^{\prime} \leq i_{2}^{\prime} \leq \ldots \leq i_{m}^{\prime} \leq i \leq t$ and $t<j_{1}^{\prime}, \ldots, j_{m}^{\prime} \leq n$. 
We have $x_{i}\left(x_{i_{1}} \ldots x_{i_{m}}\right)\left(x_{j_{1}} \ldots x_{j_{m}}\right)=\left(x_{i_{1}}^{\prime} \ldots x_{i_{m}}^{\prime}\right)\left(x_{j_{1}}^{\prime} \ldots x_{j_{m}}^{\prime}\right) x_{s}$ with $s>i$. But then,

$$
\sum_{j=1}^{i} \operatorname{deg}_{x_{j}}\left(x_{i} u_{j}\right)=m+1>m=\sum_{j=1}^{i} \operatorname{deg}_{x_{j}}\left(u_{l} x_{s}\right)
$$

which is contradiction.

In particular, by (1.1), the above lemma shows that

$$
\operatorname{depth}\left(K\left[\left\{x_{j}: j \in \mathcal{S}(I)\right\}\right] / I^{m}\right)>0 \text {, for every } m \geq 1 .
$$

First, we will identify the minimal prime ideals of $I=B_{t}(u)$, where $u=x_{i} x_{n}$ and $t+1 \leq i \leq n-t$. By applying [1, Theorem 1.1], it follows that

$$
\operatorname{Min}(I)=\left\{\left(x_{1}, \ldots, x_{i}\right)\right\} \cup\left\{\left(x_{1}, \ldots, x_{j_{1}-1}, x_{j_{1}+t}, \ldots, x_{n}\right): 1 \leq j_{1} \leq i\right\} .
$$

Let $Q$ be a monomial prime ideal associated to $I^{m}$ for some $m \geq 2$. Then $Q=Q_{A}=\left(x_{j}: j \notin A\right)$ for some set $A \subset[n]$ and $\operatorname{depth} S(Q) / I(Q)^{m}=0$, where $S(Q)=K\left[\left\{x_{j}: j \notin A\right\}\right]$ and $I(Q)$ is the localization of the ideal $I$ with respect to $Q$, that is, $I(Q)$ is obtained from $I$ by mapping the variables $x_{j} \rightarrow 1$ for $j \in A$. Therefore, in order to find all the associated monomial prime ideals of $I^{m}$ for $m \geq 2$, we need to consider the localization of $I$ with respect to some variable.

Lemma 2.3. Let $k$ be a positive integer and $P_{\{k\}}=\left(x_{j}: j \in[n] \backslash\{k\}\right)$. Let $I=B_{t}(u)$ with $u=x_{i} x_{n}, t+1 \leq i \leq n-t$, and let $k \in[n]$. Then

(1) If $k=1$, then $I\left(P_{\{k\}}\right)=\left(x_{1+t}, \ldots, x_{n}\right)$.

(2) If $1<k \leq t$, then

$$
I\left(P_{\{k\}}\right)=\left(x_{k+t}, \ldots, x_{n}\right)+\bar{B}_{t-1}\left(x_{k-1} x_{k+t-1}\right) S\left(P_{\{k\}}\right)
$$

where $\bar{B}_{t-1}\left(x_{k-1} x_{k+t-1}\right)$ is the $(t-1)$-spread principal Borel ideal generated by $x_{k-1} x_{k+t-1}$ in the polynomial ring $K\left[\left\{x_{1}, \ldots, x_{k+t-1}\right\} \backslash\left\{x_{k}\right\}\right]$.

(3) If $t<k \leq i$, then

$$
I\left(P_{\{k\}}\right)=\left(x_{1}, \ldots, x_{k-t}, x_{k+t}, \ldots, x_{n}\right)+\bar{B}_{t-1}\left(x_{k-1} x_{k+t-1}\right) S\left(P_{\{k\}}\right)
$$

where $\bar{B}_{t-1}\left(x_{k-1} x_{k+t-1}\right)$ is the $(t-1)$-spread principal Borel ideal in the polynomial ring $K\left[\left\{x_{k-1}, \ldots, x_{k+t-1}\right\} \backslash\left\{x_{k}\right\}\right]$.

(4) If $i<k<i+t$, then

$$
I\left(P_{\{k\}}\right)=\left(x_{1}, \ldots, x_{k-t}\right)+\bar{B}_{t-1}\left(x_{i} x_{n}\right) S\left(P_{\{k\}}\right)
$$

where $\bar{B}_{t-1}\left(x_{i} x_{n}\right)$ is the $(t-1)$-spread principal Borel ideal in the polynomial ring $K\left[\left\{x_{k-t+1}, \ldots, x_{n}\right\} \backslash\left\{x_{k}\right\}\right]$. 
(5) If $k \geq i+t$, then $I\left(P_{\{k\}}\right)=\left(x_{1}, \ldots, x_{i}\right)$.

Proof. Assumptions and definition of monomial localization imply that $I\left(P_{\{k\}}\right)$ for all cases, as desired.

Proof of Theorem 1.1 In order to prove the statement of the theorem, we have to show that for $m \geq 2, I^{m}$ there is no other associated prime ideal except the minimal prime ideals of $I$ and the maximal ideal. Notice that $\mathfrak{m} \in \operatorname{Ass}\left(I^{m}\right)$ for every $m \geq 2$ by $[1$, Theorem 3.1$]$.

Let $Q=Q_{A}=\left(x_{j}: j \notin A\right)$ be a monomial prime ideal which contains $I^{m}, Q \neq$ $\mathfrak{m}$. Then, $Q \in \operatorname{Ass}\left(I^{m}\right)$ if and only if $\operatorname{depth} \frac{S(Q)}{I(Q)^{m}}=0$ where $S(Q)=K\left[\left\{x_{j}: j \notin A\right\}\right]$ and $I(Q)$ is the localization of $I$ with respect to $Q$. Thus, in order to prove the desired statement, we have to show that if $Q \notin \operatorname{Min}(I)$, then $\operatorname{depth} S(Q) / I(Q)^{m}>0$.

We will distinguish the following cases.

Case (i). $Q=Q_{A} \supset\left(x_{1}, \ldots, x_{i}\right)$. Let $k=\max A$. If $k \geq i+t$, then $I(Q)=$ $I\left(P_{\{k\}}\right)=\left(x_{1}, \ldots, x_{i}\right)$. Since $Q \neq\left(x_{1}, \ldots, x_{i}\right)$, there exists $x_{l} \in Q$ with $l>i$. Thus, depth $S(Q) / I(Q)^{m}>0$ since $x_{l}$ is regular on $S(Q) / I(Q)^{m}$. Thus $Q$ is not an associated prime of $I^{m}$.

Now we assume that $k=\max A<i+t$. Obviously, we have $k \geq \min A>$ $i$. Then $Q=Q_{A} \supset\left(x_{1}, \ldots, x_{i}, x_{i+t}, \ldots, x_{n}\right)$. Then by using Lemma 2.3 , we get $I(Q)=\left(x_{1}, \ldots, x_{k-t}\right)+\bar{B}_{t-1}\left(x_{i} x_{n}\right) S(Q)$, where $\bar{B}_{t-1}\left(x_{i} x_{n}\right)$ is the $(t-1)$-spread principal Borel ideal in the polynomial ring $K\left[\left\{x_{k-t+1}, \ldots, x_{n}\right\} \backslash\left\{x_{k}\right\}\right]$. Then

$$
I(Q)^{m}=\sum_{l=0}^{m}\left(x_{1}, \ldots, x_{k-t}\right)^{m-l}\left(\bar{B}_{t-1}\left(x_{i} x_{n}\right)\right)^{l} .
$$

It is easily seen that $I(Q)^{m}$ has linear quotients with respect to decreasing pure lexicographic order. Let $G\left(I(Q)^{m}\right)=\left\{w_{1}>_{\text {lex }} \ldots>_{\text {lex }} w_{q}\right\}$ be the minimal set of generators of $I(Q)^{m}$ ordered with respect to the pure lexicographic order. Clearly, the smallest monomials in $G\left(I(Q)^{m}\right)$ are the minimal generators of $\left(B_{t-1}\left(x_{i} x_{n}\right)\right)^{m}$ ordered decreasingly with respect to the lexicographic order. By Lemma 2.2, since $i-(k-t+1)=(i-k)+(t-1)<t$, no ideal quotient of $G\left(\left(B_{t-1}\left(x_{i} x_{n}\right)\right)^{m}\right)$ contains $x_{i}$ and $x_{n}$. Therefore, by using formula (1.1) we get $\operatorname{depth} S(Q) / I(Q)^{m}>0$. This shows that $Q=Q_{A}$ is not an associated prime of $I(Q)^{m}$.

Case (ii). $Q=Q_{A} \supset\left(x_{1}, \ldots, x_{j_{1}-1}, x_{j_{1}+t}, \ldots, x_{n}\right)$ for some $j_{1} \leq i$. Then $A \subset\left[j_{1}, j_{1}+t\right]$, thus $k=\max A<i+t$ and $l=\min A \geq j_{1}$. If $l=1$, that is, $j_{1}=1$, then $I(Q)=I\left(P_{\{1\}}\right)=\left(x_{1+t}, \ldots, x_{n}\right)$, by Lemma 2.3. In this case $\operatorname{depth} S(Q) / I(Q)^{m}>0$ since $Q \supset\left(x_{1+t}, \ldots, x_{n}\right)$, thus there exists $x_{l} \in S(Q)$ which is regular on $S(Q) / I(Q)^{m}$. Let now $j_{1} \geq 2$. Then $l \geq 2$. We consider the following subcases:

(a) $i<l \leq k<i+t$

(b) $l \leq i<k<i+t$; 
(c) $l \leq k \leq i$.

In subcase (a), we get $I(Q)=I\left(P_{\{k\}}\right)$ and we derive that $\operatorname{depth} S(Q) / I(Q)^{m}>0$ as in case (i). For (b) and (c), we observe that $I(Q)$ is of the form $I(Q)=$ $\left(x_{1}, \ldots, x_{s-t}, x_{s+t}, \ldots, x_{n}, \bar{B}_{t-1}\left(x_{s-1} x_{s+t-1}\right)\right)$ for some $s$, where $\bar{B}_{t-1}\left(x_{s-1} x_{s+t-1}\right) \subset$ $K\left[\left\{x_{s-1}, \ldots, x_{s+t-1}\right\} \backslash\left\{x_{s}\right\}\right]$. Then, we order the minimal generators of $(I(Q))^{m}$ decreasingly with respect to the pure lexicographic order induced by

$$
x_{1}>\cdots>x_{s-t}>x_{s+t}>\cdots>x_{n}>x_{s-t+1}>x_{s-t+2}>\cdots>x_{s+t-1} .
$$

By a similar argument to the one used in case (i), we get $\operatorname{depth} S(Q) / I(Q)^{m}>0$ since $\bar{B}_{t-1}\left(x_{s-1} x_{s+t-1}\right)$ is a $(t-1)$-spread principal Borel ideal of the form given in Lemma 2.2. Therefore, no monomial as in Case (ii) is an associated prime of $I^{m}$.

Remark 2.4. Of course, we may consider the behavior of $\operatorname{Ass}\left(I^{m}\right)$ when $I=B_{t}(u)$ is a $t$-spread principal Borel ideal generated by $u=x_{i} x_{n}$ with $i \leq t$. To begin with, we consider $i<t$. In this case, $\mathcal{S}(I)=\bigcup_{v \in G(I)} \operatorname{supp}(v)=[n] \backslash\{i+1, i+2, \ldots, t\}$ and $I=B_{t}(u)$ is in fact an $i$-spread ideal in the polynomial ring $K\left[\left\{x_{j}: j \notin\right.\right.$ $\{i+1, i+2, \ldots, t\}\}]$. Therefore, we are reduced to considering a $t$-spread principal Borel ideal $I=B_{t}(u)$ where $u=x_{t} x_{n}$. Then we see that $I$ is the edge ideal of a bipartite graph on the vertex set $\{1,2, \ldots, t\} \cup\{t+1, t+2, \ldots, n\}$. Consequently, by [16, Theorem 5.9], I has the property that $\operatorname{Ass}\left(I^{m}\right)=\operatorname{Ass}(I)$ for all $m \geq 1$, in other words, I is normally torsion free.

\section{Arithmetical rank of principal Borel ideals generated in degree two}

In this section, we will give a direct proof of Theorem 3.2 on the arithmetic rank of a principal Borel ideals of degree 2. As we have mentioned in Introduction, we can get this result by using [12, Corollary 5.3]. A useful tool in our proof is the Schmitt-Vogel Lemma (see [15])

Lemma 3.1. Let $I \subset S$ be a squarefree monomial and $A_{1}, \ldots, A_{r}$ be some subsets of the set of monomials of I. Suppose that the following conditions hold:

(SV1) $\left|A_{1}\right|=1$ and $A_{i}$ is a finite set for any $2 \leq i \leq r$;

(SV2) The union of all the sets $A_{i}, i=1, \ldots, r$, contains the set of the minimal monomial generators of $I$.

(SV3) For any $i \geq 2$ and for any two different monomials $m_{1}, m_{2} \in A_{i}$ there exists $j<i$ and a monomial $m^{\prime} \in A_{j}$ such that $m^{\prime} \mid m_{1} m_{2}$.

Let $g_{i}=\sum_{m_{i} \in A_{i}} m_{i}$ for $1 \leq i \leq r$. Then $\sqrt{\left(g_{1}, \ldots, g_{r}\right)}=I$. In particular, $\operatorname{ara}(I) \leq r$. 
Theorem 3.2. Let $I$ be a $t$-spread principal Borel ideal, where $u=x_{i} x_{n}, i \leq n-t$. Then

$$
\operatorname{ara}(I)=\operatorname{proj} \operatorname{dim}_{S}(S / I)=n-t .
$$

Proof. By [7, Theorem 2.3] we have $\operatorname{proj}^{\operatorname{dim}_{S}}(S / I)=n-t$. We show that ara $(I)=$ $n-t$ by using the Schmitt-Vogel Lemma. We will display the minimal generators of $I$ in an upper triangular tableau as follows. In the first row, we will put the generators divisible by $x_{1}$ order decreasingly with respect to the lexicographic order. In the same manner, in the second row, we will order the monomials divisible by $x_{2}$. We shall continue this way up to the row containing the monomial divisible by $x_{i}$ where we shall put the generators $x_{i} x_{n-t} \ldots x_{i} x_{n}$. Then our tableau looks as follows.

$$
\begin{array}{rrrrrrrrr}
x_{1} x_{t+1} & x_{1} x_{t+2} & x_{1} x_{t+3} & \ldots & x_{1} x_{i+t} & \ldots & x_{1} x_{n-2} & x_{1} x_{n-1} & x_{1} x_{n} \\
& x_{2} x_{t+2} & x_{2} x_{t+3} & \ldots & x_{2} x_{i+t} & \ldots & x_{2} x_{n-2} & x_{2} x_{n-1} & x_{2} x_{n} \\
& & \ddots & & \vdots & & \vdots & \vdots & \vdots \\
& & & & x_{i} x_{t+i} & \ldots & x_{i} x_{n-2} & x_{i} x_{n-1} & x_{i} x_{n}
\end{array}
$$

Next we define the sets $A_{1}, A_{2}, \ldots, A_{n-t}$ in the following way. In the first set, we will put the monomial from the right up corner of the tableau. In the second set, we will put the two monomials from the right up parallel to the diagonal of triangular tableau. In the third set, we will collect the three monomials from the next parallel to the diagonal, and so on. Explicitly, the sets are the following ones.

$$
\begin{aligned}
& A_{1}=\left\{x_{1} x_{n}\right\} \\
& A_{2}=\left\{x_{1} x_{n-1}, x_{2} x_{n}\right\} \\
& A_{3}=\left\{x_{1} x_{n-2}, x_{2} x_{n-1}, x_{3} x_{n}\right\} \\
& \vdots \\
& A_{j}=\left\{x_{1} x_{n-j+1}, x_{2} x_{n-j+2}, \ldots, x_{j} x_{n}\right\}, \text { for } i \geq j \\
& \vdots \\
& A_{j}=\left\{x_{1} x_{n-j+1}, x_{2} x_{n-j+2}, \ldots, x_{i} x_{n-j+i}\right\}, \text { for } i<j \\
& \vdots \\
& A_{n-t-1}=\left\{x_{1} x_{t+2}, x_{2} x_{t+3}, \ldots, x_{i} x_{i+t+1}\right\} \\
& A_{n-t}=\left\{x_{1} x_{t+1}, x_{2} x_{t+2}, \ldots, x_{i} x_{i+t} .\right\}
\end{aligned}
$$

One may easily check that the sets $A_{1}, \ldots, A_{n-t}$ verify all conditions of the Schmitt-Vogel Lemma. The first two conditions of Lemma 3.1 are clearly fulfilled. We hall sgive an explanation for the third condition only. If we pick up two different monomials in the set $A_{j}$ for some $j \geq 2$, let us say $m_{1}$ from the $k$-th row and $m_{2}$ from the $l$-th row of the tableau with $k<l$, then we put the monomial which is the intersection element of the $k$-th row and the column of $m_{2}$ as $m^{\prime}$ which divides the product $m_{1} m_{2}$ and $m^{\prime} \in A_{r}$ for some $r<j$. For instance, if $m_{1}=x_{k} x_{n-j+k}, m_{2}=$ $x_{l} x_{n-j+l} \in A_{j}$ for some $k<l$ then we choose $m^{\prime}=x_{k} x_{n-j+l} \in A_{k+j-l}$ which divides $m_{1} m_{2}=x_{k} x_{l} x_{n-j+k} x_{n-j+l}$. 
We recall from [2] that the ideal $I$ is called a set-theoretic complete intersection if height $(I)=\operatorname{ara}(I)$. An ideal $I$ is called cohomologically complete intersection if $h t(I)=c d(I)$.

Proposition 3.3. Let $I=B_{t}(u)$ be a t-spread principal Borel ideal generated in degree 2 . Then $I$ is a set theoretic compete intersection if and only if $u=x_{n-t} x_{n}$.

Proof. Let $u=x_{i} x_{n}$. By Theorem 3.2, we have $\operatorname{ara}(I)=\operatorname{proj} \operatorname{dim}(S / I)=n-t$. By $[1$, Theorem 1.1], we know that height $(I)=i$. Thus height $(I)=\operatorname{ara}(I)$ if and only if $i=n-t$.

Proposition 3.4. Let $t \geq 1$ be an integer and $I_{n, d, t} \subset S$ the $t$-spread Veronese ideal generated in degree $d$. Then I is a cohomologically complete intersection ideal. In particular, $\operatorname{cd}\left(I_{n, d, t}\right)=n-t(d-1)$.

Proof. By [7, Theorem 2.3], $I$ is Cohen-Macaulay and $\operatorname{cd}\left(R, I_{n, d, t}\right)=\operatorname{height}\left(I_{n, d, t}\right)=$ $n-t(d-1)$. So $I_{n, d, t}$ is cohomologically intersection.

\section{Lyubeznik numbers}

Suppose that $(R, m, K)$ is a local ring admitting a surjection from an $n$-dimensional regular local ring $(S, n, K)$ containing a field, and let $I$ denote the kernel of the surjection. Given $i, j \in \mathbb{N}$, the Lyubeznik number of $R$ with respect to $i, j \in \mathbb{N}$, is defined as

$$
\lambda_{i, j}(R)=\operatorname{dim}_{K} \operatorname{Ext}_{S}^{i}\left(K, H_{I}^{n-j}(S)\right)
$$

and is denoted $\lambda_{i, j}(R)$. Put $d=\operatorname{dim} R$, Lyubeznik numbers satisfy the following properties:

(a) $\lambda_{i, j}(R)=0$ for $j>d$ or $i>j$.

(b) $\lambda_{d, d}(R) \neq 0$.

(c) If $R$ is Cohen-Macaulay, then $\lambda_{d, d}(R)=1$.

(d) Euler characteristic,

$$
\sum_{0 \leq i, j \leq d}(-1)^{i-j} \lambda_{i, j}(R)=1 .
$$

Therefore, we can record all nonzero Lyubeznik numbers in the so-called Lyubeznik table: 


$$
\left[\begin{array}{ccccc}
\lambda_{0,0} & \cdot & \cdot & \cdot & \lambda_{0, d} \\
0 & \cdot & & & \cdot \\
0 & 0 & \cdot & & \cdot \\
0 & 0 & 0 & \cdot & \cdot \\
0 & 0 & 0 & 0 & \lambda_{d, d}
\end{array}\right]
$$

where $\lambda_{i, j}:=\lambda_{i, j}(R)$ for every $0 \leq i, j \leq d$, see for example [2].

Corollary 4.1. Lyubeznik table of $I_{n, d, t}=J \subset S$ is

$$
\lambda_{i, j}(S / J)=0 \text { for all } 0 \leq i, j<d \text { and } \lambda_{d, d}=1,
$$

where $\operatorname{dim}(S / J)=d$.

Proof. [7, Theorem 2.3].

Lemma 4.2. Let $S=k\left[x_{1}, \ldots, x_{n}\right]$ be a polynomial ring over a field $k, m$ which denotes its homogeneous maximal ideal $\left(x_{1}, \ldots, x_{n}\right)$ and $I=B_{t}(u)$ where $u=$ $x_{n-t} x_{n}$. Then

$$
\lambda_{i, j}(S / I)=0 \text { for all } 0 \leq i, j<d \text { and } \lambda_{d, d}=1 \text {. }
$$

Proof. As $I$ is cohomologically complete intersection,

$$
\operatorname{dim}(S / I)=\operatorname{fgrade}(I, S) .
$$

So

$$
\operatorname{depth}(S / I) \leq \operatorname{fgrade}(I, S) .
$$

By $[2$, lemma 3.2] we conclude that

$$
\lambda_{i, j}(S / I)=0 \text { for all } 0 \leq i, j<d \text { and } \lambda_{d, d}=1 .
$$

\section{REF E R E N C E S}

1. C. Andrei and V. Ene, B. Lajmiri : Power of t-spread principal Borel ideals. Archiv der Mathematik, 113 (2018), 1420-8938.

2. Kh. Ahmadi-Amoli, E. Banisaeed and M.Eghbali, and F. Rahmati: On the relation between formal grade and depth with a view toward vanishing of Lyubeznik numbers. Communications in Algebra, 45(2017), 5137-5144.

3. M. BARILE: On the arithmetical rank of the edge ideals of forests. COMm. AlgeBra, 36(2008), 4678-4703.

4. M. Brodmann: Asymtotic stablitiy of $\operatorname{Ass}\left(M / I^{n} M\right)$. Proc. Am. Math. Soc, 74 (1979), 16-18. 
5. M. BARILE and N. Terai: Arithmetical ranks of Stanley-Reisner ideals of simplicial complexes with a cone. Comm. Algebra, 38 (2010), 3686-3698.

6. J. M. Bernal, S. Morey and R. H. Villarreal: Associated primes of powers of edge ideals. Collect. Math. 63 (2012), 361-374.

7. V. Ene, J. Herzog and A. Asloob QUREshi: t-spread strongly stable monomial ideals. Communications in Algebra, (2019), 1-14.

8. V. Ene, O. Olteanu and N. Terai : Arithmetical rank of lexsegment edge ideals. Bull. Math. Soc. Sci. Math. Roumanie (N.S.), 53 (2010), 315-327.

9. J. Herzog and T. Hibi : Monomial ideals. Grad. Texts in Math, 260, SPRINGER, LONDON, 2010.

10. J. Herzog and A. Asloob Qureshi : Persistence and stability properties of powers of ideals. J. Pure Appl. AlgeBra, 219 (2015), 530-542.

11. J. Herzog, A. RAUf and M. VlădoIU: The stable set of associated prime ideals of a polymatroidal ideal. J. Algebraic Combin, 37 (2013), 289-312.

12. K. Kimura : Arithmatical rank of Cohen-Macaulay squarefree monomial ideals of height two, J. Commut.Algebra., 3 (2011), 31-46.

13. K. Kimura, N. Terai and K. Yoshida: Arithmetical rank of squarefree monomial ideals of small arithmetic degree. J. Algebraic Combin, 29 (2009), 389404.

14. G. LyubezniK : On the local cohomology modules $H_{\mathfrak{a}}^{i}(R)$ for ideals a generated by monomials in an R-sequence. SPRINGER-VERLAG, 1092 (1984), 214-220.

15. T. Schmitt and W. Vogel: Note on set-theoretic intersections of subvarieties of projective space. MATH. AnN, 245 (1979), 247-253.

16. A. Simis, W. Vasconcelos and R. H. Villarreal: On the Ideal Theory of Graphs.J. AlgeBra, 167 (1994), 389-416.

Bahareh Lajmiri Amirkabir University of Technology

Department of Mathematics and Computer Science

424 Hafez Ave, Tehran, Iran

bahareh.lajmiri@aut.ac.ir

Farhad Rahmati Amirkabir University of Technology

Department of Mathematics and Computer Science

424 Hafez Ave, Tehran, Iran

frahmati@aut.ac.ir 